\title{
Epidemiological dynamics of an urban Dengue 4 outbreak in São Paulo, Brazil
}

Christian Julián Villabona-Arenas, Jessica Luana de Oliveira, Carla de Sousa-Capra, Karime Balarini, Celso Ricardo Theoto Pereira da Fonseca, Paolo M de A Zanotto

Background Dengue studies at the urban scale are scarce and required for guiding control efforts. In Brazil, the burden of dengue is high and challenges city public health administrations with limited resources. Here we studied the dynamics of a dengue epidemic in a single city. Methods Serum samples from dengue suspected cases were collected and tested, from December 2012 and July 2013 in Guarujá, Brazil. We use incidence series analysis to provide a detailed view of the reproduction number dynamics and a Bayesian analysis to infer the spread of the serotype using geographic and temporal data. Results We obtained nucleotide sequences from 354 envelope genes and georeferenced 286 samples during the course of the outbreak. Serotype 4 was responsible for the epidemic. We identified at least two major lineages that overlapped in distribution. We observed high Reproduction numbers and high cladogenesis prior to the escalation of clinical case notifications. Three densely populated non-adjacent neighborhoods played a pivotal role during the onset and/or course of the epidemic. Discussion Our findings point to high dengue virus transmission with a substantial proportion of unapparent cases that led to a late recognition of an outbreak. Usually source reductions initiatives tend to be insufficient once an epidemic has been established. Nevertheless, health authorities in Guarujá prioritized vector control on specific places with clusters of georeferenced viremic patients, which appear to have diminished the epidemic impact. 
1 Epidemiological dynamics of an urban Dengue 4 outbreak in São

2 Paulo, Brazil

3

4 Authors

5 Christian Julián Villabona-Arenas ${ }^{1}$, Jessica Luana de Oliveira ${ }^{1,2}$, Carla de Sousa-Capra $^{3}$,

6 Karime Balarini ${ }^{4}$, Celso Ricardo Theoto Pereira da Fonseca ${ }^{4}$, Paolo Marinho de Andrade

7 Zanotto $^{1}$

8

9 Affiliations

$10{ }^{1}$ Laboratory of Molecular Evolution and Bioinformatics, Department of Microbiology,

11 Biomedical Sciences Institute, University of São Paulo, São Paulo, Brazil.

$12{ }^{2}$ Department of Biomedicine, University of Mogi das Cruzes, Mogi das Cruzes, São Paulo,

13 Brazil

$14{ }^{3}$ Office of Epidemiological Surveillance, Department of Health, Guarujá, São Paulo, Brazil.

$15{ }^{4}$ ITAPEMA, clinical laboratory analysis center, Guarujá, Brazil.

16

$17 *$ Corresponding author:

18 Paolo Marinho de Andrade Zanotto

19 Av. Prof. Lineu Prestes, 1374, Sao Paulo, SP, 05508-000, Brazil

20 pzanotto@usp.br

21

22 


\section{Abstract}

\section{Background}

25 Dengue studies at the urban scale are scarce and required for guiding control efforts. In

26 Brazil, the burden of dengue is high and this challenges city public health administrations

27 with limited resources. Here we studied the dynamics of a dengue epidemic in a single city.

\section{Methods}

29 Serum samples from dengue suspected cases were collected and tested, from December

302012 to July 2013 in the city of Guarujá, Brazil. We use incidence series analysis to provide a

31 detailed view of the reproduction number dynamics and a Bayesian analysis to infer the

32 spread of the serotype using geographic and temporal data.

\section{Results}

34 We obtained nucleotide sequences from 354 envelope genes and georeferenced 286

35 samples during the course of the outbreak. Serotype 4 was responsible for the epidemic.

36 We identified at least two major lineages that overlapped in distribution. We observed high

37 Reproduction numbers and high cladogenesis prior to the escalation of clinical case

38 notifications. Three densely populated non-adjacent neighborhoods played a pivotal role

39 during the onset and/or course of the epidemic.

40 Discussion

41 Our findings point to high dengue virus transmission with a substantial proportion of

42 unapparent cases that led to a late recognition of an outbreak. Usually source reductions

43 initiatives tend to be insufficient once an epidemic has been established. Nevertheless,

44 health authorities in Guarujá prioritized vector control on places that concentrated

45 georeferenced viremic patients, which appear to have diminished the epidemic impact. 


\section{Introduction:}

48

The dengue viruses exist as four antigenically distinct serotypes named DENV-1,

DENV-2, DENV-3 and DENV-4. Dengue fever (DF) is a disease caused by any of the DENV

51 (Chen \& Vasilakis 2011). There is an estimate of 390-million (95\% credible interval 284-528)

52 dengue infections worldwide per year, of which 96 million (67-136) show any level of

53 clinical or subclinical severity (Bhatt et al. 2013).

during the wet season when Aedes mosquitoes' population sizes are high and the rainfall is optimal for breeding. People provide the mosquitoes not only with blood meals but also

water-holding containers where the mosquitoes lay their eggs. In addition, this country is at periodic risk for epidemic dengue (i.e., when large numbers of people become infected during a short period), which requires a coincidence of large numbers of vector mosquitoes and large numbers of people with no immunity to one or more of the four serotypes (CDC 2015). after it was last detected in the country in 1982, and has been responsible for several outbreaks since then (Nunes et al. 2012). In 2013, 1,468,873 million dengue cases were reported countrywide, including 6,969 severe cases and 545 deaths. These numbers entail a challenge for public health authorities, which in a timely manner need to allocate resources and trained personnel to try diminishing the health impact of the disease. Programs to control populations of mosquitoes strain public resources, especially in resource-limited settings (Shepard et al. 2011; Stahl et al. 2013). In this context, understanding epidemic 
70 spread in urban settings is crucial because the results may guide the allocation of scarce

71 resources toward future vector control.

72 The spatiotemporal patterns of dengue spread in Brazilian settings are limited and

73 mostly based on serological prevalence and incidence data (Barreto \& Teixeira 2008;

74 Teixeira Mda et al. 2002; Teixeira et al. 2013). Some recent studies address this topic in a

75 larger scale (Nunes et al. 2012; Nunes et al. 2014). So far, only one work addresses the

76 spatial dynamics of an urban dengue outbreak in the city of São Jose de Rio Petro using viral

77 genetic data (Mondini et al. 2009). These studies are imperative because socio-demographic

78 and ecological factors affect diffusion dynamics (Cuong et al. 2013; Jeefoo et al. 2011;

79 Raghwani et al. 2011; Rasmussen et al. 2014; Schreiber et al. 2009; Vazquez-Prokopec et al.

80 2010). In the present work we describe an outbreak of DENV-4 during 2013 in the city of

81 Guarujá, Brazil, following Bayesian phylogenetic analysis of envelope gene sequences. Our

82 results emphasize the importance of real-time follow up and guided actions to achieve

83 better control during epidemics.

84

85 Methods:

86

87 Study Site

88

Guarujá $\left(23^{\circ} 59^{\prime} 37^{\prime \prime} S 46^{\circ} 15^{\prime} 23^{\prime \prime} \mathrm{W}\right)$ is a coastal city in Santo Amaro Island,

90 situated at the shore of the State of São Paulo, Brazil (Fig. 1A). The city is embedded in a

91 tropical rain forest. It has a tropical humid climate that is characterized by having high

92 average air temperature and rainfall. The average annual temperature is $24.7^{\circ} \mathrm{C}$ ( $\mathrm{Min} 18^{\circ} \mathrm{C}$

93 Max $31.3^{\circ} \mathrm{C}$ ) and the annual rainfall is 3,400 $\mathrm{mm}$; February is the wettest month (average 
94 rainfall of $413 \mathrm{~mm}$ ) and August is the driest one (average rainfall of $156 \mathrm{~mm}$ ). The city main

95 economic sources are seasonal tourism and port related activities. The estimated

96 population in 2013 was 306,683 and the human population density was around 2,000

97 inhabitants per $\mathrm{km}^{2}$. Official dengue figures by the Epidemiological Surveillance Center of

98 the State (CVE) date back to 1997 and sum 24000 total cases up to 2012. There is no

99 detailed information concerning the previous exposure to distinct DENV serotypes and the

100 municipality relies on the surveillance by the Adolfo Lutz Institute (The Central Public Health

101 laboratory from The State of São Paulo).

102

103 Sample Collection:

104

105

In late 2012, our group at University of São Paulo joined efforts with the Guarujá

106 Municipality's office of epidemiological surveillance and with a local clinical laboratory

107 analysis center (Itapema) to map the incidence of dengue in the city and obtain viral genetic

108 data. Both institutions contributed with the collection of samples citywide and by

109 performing preliminary immunochromatographic diagnostic tests for dengue (Kassim 2011).

110 Patients of any age with symptoms and signs of dengue disease that were examined

111 in Primary Health or Emergency Care Units and tested positive for IgM and/or NS1 were

112 considered for the study. Symptoms included fever, frontal or retro-orbital headache,

113 severe pain (muscles, bones, legs, joints, lower back or abdominal), nausea, vomiting, taste

114 disturbance and anorexia. Signs included high fever (usually between 38.5 and $41^{\circ} \mathrm{C}$ )

115 persisting for 24 hours, rash, hemorrhagic manifestations, hypotension and narrow pulse

116 pressure.

117 
118 Ethics Statement

119

120 The Ethical Review Board of the Biomedical Science Institute at University of São

121 Paulo approved this study (Statement 933/CEP). All adult subjects provided an informed

122 written consent, and a parent or guardian of any child participant provided the written

123 informed consent on their behalf.

124

125 Molecular Testing

126

Viral RNA was extracted from serum samples with the QIAmp viral RNA mini kit

128 (Qiagen, Venlo, Limburg, Netherlands) and the complementary DNA was synthesized using

129 the SuperScript ${ }^{\circledR}$ VILO ${ }^{\text {TM }}$ cDNA Synthesis Kit (Life Technologies, Carlsbad, California, United

130 States). The GoTaq ${ }^{\circledR}$ Green Master Mix (Promega, Madison, Wisconsin, United States) was

131 used for PCR amplifications of the envelope gene using the primers of (Bennett et al. 2003).

132 The ExoSAP-IT reagent was used for PCR Product Cleanup (Affymetrix, Santa Clara, California,

133 United States) and the sequencing reaction was performed using the BigDye ${ }^{\circledR}$ Terminator

134 v3.1 Cycle Sequencing Kit (Life Technologies). Sequencing reaction products were purified

135 using the BigDye XTerminator Purification Kit (Life Technologies) and sequenced on an ABI

$136 \mathrm{PRISM}^{\circledR} 3130$ Genetic Analyzer (Life Technologies). Contigs were assembled using the

137 program Codon Code aligner.

138

139 Genetic Analysis

140 
142 visual inspection and manual editing with Mesquite 2.75 (Maddison \& Maddison 2014).

143 Polymorphisms were analyzed with DNASP 5 (Librado \& Rozas 2009). The Tajima D statistic

144 test was used to evaluate deviations from the neutral expectation of molecular evolution

145 (Tajima 1989). The package HyPhy v2.2 was used to screen for recombination (SBP-Single

146 Breakpoint Recombination and GARD-Genetic Algorithms for Recombination Detection) and

147 for positive selection; the dengue strain H780090 isolated in Boa Vista, RR-Brazil (29 of

148 November of 2010) was used as a reference for selection analysis. Both genealogy-based,

149 codon-site models Single Likelihood Ancestor Counting (SLAC) and the Fixed Effects

150 Likelihood (FEL) methods were used to estimate the non-synonymous ( $\mathrm{dN}$ ) and synonymous

151 (dS) rates of substitution (Delport et al. 2010; Kosakovsky Pond \& Frost 2005; Pond et al.

152 2005).

153

154 Phylogenetic analysis

155

The sequences obtained in this study were combined with a DENV-4 database from a

157 previous study (Villabona-Arenas \& Zanotto 2011) to identify the genotype. This was

158 achieved using high-throughput clustering with the UCLUST algorithm in the package

159 USEARCH (Edgar 2010).

160 The JModeltest software was used for the statistical selection of the best-fit model

161 of nucleotide substitution under the Akaike information criterion (Darriba et al. 2012;

162 Guindon \& Gascuel 2003). Sequences were dated according to the day of sampling and used

163 for phylogenetic reconstruction and the estimation of the rate of evolutionary change $(\mu)$

164 (subs/site/year) using Bayesian Inference (IB) in Beast v2.3.1 (Bouckaert et al. 2014); the 
165 tree prior was a Birth-Death with Serial Skyline Sampling (BDSKY) (Stadler et al. 2013) (See

166 Table S1 for parameterization). A Bayesian maximum clade credibility (MCC) tree was

167 inferred from a set of plausible trees sampled at the stationary phase of four independent

168 Markov Chain Monte Carlo (MCMC) runs with 200 million generations each using a relaxed

169 (uncorrelated lognormal) molecular clock (Drummond et al. 2006). The convergence of

170 parameters was assessed using Tracer v1.6 program

171 (http://tree.bio.ed.ac.uk/software/tracer/) until all parameters estimates showed Effective

172 Sample Size (ESS) values over 200.

173

174 Time-varying reproduction numbers

175

176

We used the approach of Cori et al. to estimate the instantaneous reproduction

177 number using the R-package EpiEstim (Cori et al. 2013; Salje et al. 2012). The method

178 requires incidence data and a serial interval distribution (a gamma distribution with shift 1)

179 that describes the time between the onset of symptoms in a primary case and the onset of

180 symptoms of secondary cases. We used the weekly notifications from the municipality's

181 office of epidemiological surveillance for the epidemiological year of 2012-2013 (daily data

182 was not available) and a serial interval distribution that reflected previous estimates of the

183 dengue incubation period (Table S1); the outcome of a censored Bayesian time-to-event

184 model estimated the dengue intrinsic incubation period (the time between a human being

185 infected and the onset of symptoms due to the infection) around six days ( $95 \% \mathrm{Cl} 3-10$

186 days) and the best-fitting temperature-dependent extrinsic incubation period around 6.5

187 days ( $95 \% \mathrm{Cl} 2-15$ days) at $30^{\circ} \mathrm{C}$ (Chan \& Johansson 2012). The analysis took into account the

188 uncertainty in the serial interval distribution by integrating over a range of means and 
189 standard deviations of the serial interval (the mean and standard deviation were allowed to

190 vary according to truncated normal distributions, Table S1) (Cori et al. 2013). We used a

191 gamma prior distribution (Table S1) for the reproduction number that includes previous

192 estimates (between 1.33 and 11.6) (Halstead 2008).

193

194

195 Spatiotemporal dispersion pattern

196

197

In order to find the most parsimonious set of rates explaining the diffusion process

198 along the sampled trees for a geolocated dataset, we used the Bayesian stochastic search

199 variable selection (BSSVS) approach as implemented in Beast v2.3.1 (Bouckaert et al. 2014).

200 The method assumes exchange rates in a continuous-time Markov chain (CTMC) to be zero

201 with some prior probability (Lemey et al. 2009) and performs ancestral reconstruction on a

202 single character, which represents the location of the taxa. Sampled taxa are associated

203 with locations and the ancestral states of the internal nodes in the sampled trees can be

204 reconstructed from the taxon locations; because the sampled trees are in units of time we

205 can reconstruct the diffusion over time by following character transformation over the

206 branches. Locations were represented by discrete groups of adjacent neighborhoods

207 (discrete phylogeography) and a Bayes factor (BF) test was run to identify the rates

208 contributing to the migration path with the software Spread v1.0.4 (Bielejec et al. 2011).

209 The number of neighborhoods was reduced to a maximum of 10 localities (chosen by

210 vicinity a by number of samples reported) in order to diminish sample-size bias

211 (disproportionate sampling can strongly bias phylogeographic analyses because over

212 sampled populations will more likely to be inferred as source populations). 


\section{Results:}

216 Sampling

The year 2013 coincided with a steep rise in the confirmed cases of dengue fever in

219 the State of São Paulo (Fig. 1B). Public Health authorities of Guarujá reported a total of 1805 autochthonous dengue cases during this year.

We studied 505 PCR dengue-positive patients during the study In Guarujá. These samples were collected between December 2012 and July 2013. Serotyping determined 10

$223(1.9 \%)$ to be DENV-1, eight (1.5\%) to be DENV-2, two to be DENV-3 $(0.4 \%)$ and 505 to be DENV-4 (96.2\%). DENV-4 was relatively new to the country and outbreaks had been reported throughout the country since it was first detected in Brazil in 2011. Preliminary results reporting the documentation of the co-circulation of the four serotypes was

227 published elsewhere (Villabona-Arenas et al. 2014).

230 yield sufficient viral RNA). These sequences were deposited in GenBank under the accessions KP703864 - KP704217.

DENV-4 genetic diversity

236 fell in the first and second codon position and $77(5.2 \%)$ fell in the third codon position. 
237 There were a total of 95 haplotypes and 75 of them represented one unique sequence; the

238 three most frequent haplotypes had 119 (34\%), $38(11 \%)$ and $30(8.5 \%)$ sequences

239 (Haplotype diversity, the probability that two haplotypes drawn uniformly at random from

240 the population are not the same, was 0.86$)$. The Tajima's D value was of $-2.56(P<0.001)(-$

2412.48 for the combination of both first and second codon positions, -2.26 for the third codon

242 position) evidenced an excess of low frequency polymorphisms relative to expectation,

243 indicating population size expansions and/or purifying selection. In agreement with this, the

244 overall rate of non-synonymous over synonymous changes $d N / d S$ value of $0.15(95 \% \mathrm{Cl}$

245 0.10-0.21) for the entire gene suggested purifying selection. A few codons, which fell in the 246 central and dimerization gene domains, showed statistically significant purifying selection

247 (codons 92, 133, 184 and 225) at the significant level of 0.05. Although a few sites

248 experienced an elevation on $d N / d S$ there was no statistical evidence for adaptive evolution.

250 Evolutionary history and epidemiological dynamics

The 354 DENV-4 local sequences fell into the Latin-American cluster of viruses of

253 Genotype II together with other Brazilian samples (see Data S1).

sites. The mean evolutionary rate was $2.79 \times 10^{-3}$ substitutions per site per year (95\% HPDs: parameters were: the sampling proportion was $1.4 \%$ (95\% HPDs: $0.03-3.1 \%)$, the infectious period was 6.7 days (95\% HPDs: 5-10 days) and the origin of the epidemic was the 21th of

259 December-2012 (95\% HPDs: $16^{\text {th }}$ December-2012 $-26^{\text {th }}$ December-2012). Figure S1 show

260 the extent to which prior information matched the posterior. Sampling from the prior 
261 analysis indicated that the posterior and prior traces were the same and that the overall

262 constraints were not forcing the results.

263 Fig. 2A shows the MCC tree with two clades early on in the epidemic. The mean time

264 to the most recent common ancestor of these clades did not differ significantly, suggesting

265 that both viral lineages diverged over similar time-scales, and then co-circulated.

267 indicates that the disease will be able to spread in a population. For the time series analysis,

268 the estimates are high during the first four months of the year and the curve decrease

269 rapidly by the end of April with values below 1.0 in May and June. We did not included the

270 birth-death skyline plots because this reconstruction may be misleading around the time we

271 had severe sampling issues (due to the switch to clinical diagnosis) and not enough

272 phylogenetic diversity was observed within the sampled genetic data (du Plessis \& Stadler

273 2015); additional BDSKY analyses where sampling proportions were estimated in a piece-

274 wise manner over six different intervals did not solve this. Moreover, these methods not

275 always reconstruct complex dynamics when other factors such as seasonality, spatial

276 structure and vector dynamics are not incorporated (Rasmussen et al. 2014)

278 Phylogeography of DENV-4 over the city

We were able to geolocate 286 patients (81\%) (Fig. 1A) based on the addresses recorded by the Guarujá Municipal Health Department (Records were not available for the

282 remaining patients); Figure 2B compares the actual number of official cases reported and our DENV-4 geolocated sampling. Our first geolocated sample was collected in January the

2842 nd 2013 at the neighborhood Enseada. This location has a high number of residents 
285 (20.883 based on the 2010 census records) and is home to the largest beach concentrating

286 mostly residents. Pae-Cará together with its neighbor Itapema, are the neighborhoods with

287 more residents (26,054 and 26,070 respectively) followed by Morrinhos (24,387), Enseada

288 and Jardim Boa Esperança $(20,753)$. The digital map was provided by the Municipality's

289 office and represents the master plan for development and urban planning in the city.

290 Figure 3 illustrates the overall discrete spatial diffusion over the urban area. These results

291 were gauged from a full location-annotated MCC tree, available as Fig. 4, which evidenced

292 an early widespread distribution of the virus in January. The introduction events into each

293 discrete unit are depicted in Fig. 3A; these represent viral diffusion during the onset of the

294 epidemic. These figures suggest that two localities, Enseada and Pae-Cará, were key virus

295 sources. Later on, all regions become interconnected in terms of viral diffusion. The

296 adjacent high-income, low-population density neighborhoods (Jardim Acapulco and

297 Pernambuco) had no cases sampled. This is not explained by distance or lack of connection,

298 because low-income areas nearby (the shantytowns in Mar e Céu, to the South, and

299 Pereque Beach, to the North) had several cases during the epidemic. Bayes factor test of

300 significant diffusion rates shows that another two localities (Morrinhos and Jardim Boa

301 Esperança) played an important role during the course of the epidemic (Fig. 3B). The initial

302 diffusion pattern reproduced to some degree the main access highways of the island: a

303 north-south axis with Pae-Cará and a west-east axis over the littoral with Enseada. (Fig. 3C)

304

305

Discussion:

306

307 In the present study we described the outbreak of DENV-4 during 2013 in the city of Guarujá,

308 Brazil. During the outbreak we documented purifying selection and found no statistical 
309 evidence of adaptive evolution. Nonetheless, inferences about selection and rates drawn

310 from the analyses should be interpreted with caution. The observed differences between

311 our sequences may represent segregating sites in a population and under this scenario

312 evolutionary rates can be overestimated and $\mathrm{dN} / \mathrm{dS}$ ratios below one can be found under

313 both negative and positive selection (Kryazhimskiy \& Plotkin 2008).

314 On April 4th, the Public health authorities of Guarujá announced the epidemic alert

315 (when a city reaches the incidence of 100 cases per 100 thousand inhabitants) and during

316 this month they strengthened control measures. Confirmatory diagnoses for dengue

317 became clinical (ignoring the possibility of other acute febrile illnesses) and the number of 318 cases increased dramatically after the epidemic alert was announced.

The reproduction number dynamics (Fig $2 \mathrm{~B}$ ) and the timing of the coalescent events

320 (concentrated around February) point to an epidemic that started much earlier than the

321 case report records. A comparable observation was done in another Brazilian setting

322 (Mondini et al. 2009): the epidemic peak by demographic skyline methods took place

323 around two months before the epidemic peak by case report data. The authors argue that

324 such finding resulted from an increase in false positives after the epidemic alert and we

325 argue that in Guarujá the population awareness might have also contributed to a sudden

326 increase.

Higher values in reproduction numbers (or relative genetic diversity estimates)

328 preceding the peak of laboratory-confirmed cases may reflect virus population spread in a

329 large unreported infected population. Dengue virus infection results in more asymptomatic

330 cases than symptomatic ones and this difficult the early detection of increased incidence.

331 This phenomenon has been documented for Brazilian urban settings (Endy 2002; Poblap et

332 al. 2006; Teixeira Mda et al. 2002). The spatial diffusion analysis shows that when a 
333 significant number of clinical cases began to appear, the virus was practically distributed

334 throughout the city (Fig. 4). Moreover, by looking at the available data on dengue from 2012

335 (Fig. 1C) it is clear that transmission is sustained all over the year. Following the dynamics of

336 the reproduction number using incidence time series, we observed that the disease spread

337 quickly during most of the observational period and decreased by the end.

338 During November of 2012, the city led the ranking of mosquito infestation in the

339 State of São Paulo and the averaged Breteau index for Aedes aegypti during 2103 was high

340 (3.12). Source reduction initiatives started in shantytowns during March but the flow of

341 people and of mosquitoes from other areas may have offset their contribution. Municipality

342 health authorities considered the use of massive insecticide nebulization when extensive

343 symptomatic cases were recognized. The use of mosquito fogging trucks is not executed

344 citywide because it is costly and relies on availability from the Adolfo Lutz Institute,

345 therefore strategic areas have to be selected. The neighborhoods of Enseada, Paé-Cara and

346 Morrinhos were selected because they reported higher numbers of reactive NS1 antigen

347 results; four rounds of insecticide nebulization were applied during April and massive

348 insecticide nebulization coincided with a rapid reduction in the number of cases over time.

349 It may be argued that the outcome was due to the depletion of the susceptible population

350 or only a change in seasonality(Egger et al. 2008). Nonetheless, Guarujá was among the

351 municipalities (103 out of 429) that showed a statistically significant reduction of

352 notification when comparing the figures of the previous epidemics (2010) and those of the

353 State (a naïve-experienced population to DENV-4). Overall, this suggests that control

354 strategy achieved some degree of control. 
The research into their effectiveness of vector control is scarce and almost nothing is

357 known about how well it reduces DENV transmission (Achee et al. 2015). The two high-

358 income neighborhoods that had privately-owned and hired vector control services (as

359 informed by the health authorities) showed a discontinuity when pinpointing the dengue

360 incidence over the city. This suggests that control actions, when applied in a timely and

361 sustained manner, are useful. Rapid and unplanned urbanization (e.g. shantytowns) has

362 provided appropriate circumstances (high population density and high contact rates

363 between humans and mosquitos) for substantial vector breeding in Guarujá and several

364 municipalities of Brazil. For example, nearby cities, such as Santos and São Vicente (Fig 1B),

365 also have important number of dengue notifications throughout the year and therefore a

366 continuous flux of infected people and mosquitoes is expected. Under this scenario, city

367 public health administrations with limited resources encounter a big challenge. In Guarujá,

368 local authorities prioritized vector control based on georeferenced viremic patients and the

369 epidemic of an expected higher magnitude. To achieve full control however, this may be not 370 enough, but it is a first coherent step.

372 Conclusion:

Studying urban outbreaks is important; successful public health interventions require

375 detailed knowledge of the disease dynamics and how it spread within the population. It is

376 very difficult to stop dengue spread because Aedes mosquitoes bounce back to initial

377 numbers after control interventions and because unapparent infections also contribute to

378 DENV persistent circulation (Duong et al. 2015). Nonetheless, in the absence of a vaccine,

379 source reduction initiatives and massive control actions are the options that city public 
380 health administrations have. We have evidenced that a delayed response may result in an

381 epidemic that grow beyond the capabilities of local health authorities but that sound efforts

382 may diminish its effect.

383

384 Acknowledgments:

385

386

We thank all the participants in this study for generously helping us in this research.

387

We also thank all the medical staff and administrative staff from the Primary Health Care

388

Facilities, the Emergency Care Units and the ITAPEMA clinical laboratory analysis center who

have kindly assisted us by collecting blood samples from participants. We thank the Guarujá

Municipality's office of epidemiological surveillance for the discussions and the practical and

technical advice.

References:

Achee NL, Gould F, Perkins TA, Reiner RC, Jr., Morrison AC, Ritchie SA, Gubler DJ, Teyssou R, and Scott TW. 2015. A critical assessment of vector control for dengue prevention. PLoS Negl Trop Dis 9:e0003655. 10.1371/journal.pntd.0003655

400

401

402

403

404

405

406

407

408

409

410

Barreto ML, and Teixeira MG. 2008. Dengue in Brazil: Epidemiological situation and Contribution to a Research Agenda. estudos avançados 22:53-72.

Bennett SN, Holmes EC, Chirivella M, Rodriguez DM, Beltran M, Vorndam V, Gubler DJ, and McMillan WO. 2003. Selection-driven evolution of emergent dengue virus. Mol Biol Evol 20:1650-1658. 10.1093/molbev/msg182

Bhatt S, Gething PW, Brady OJ, Messina JP, Farlow AW, Moyes CL, Drake JM, Brownstein JS, Hoen AG, Sankoh O, Myers MF, George DB, Jaenisch T, Wint GR, Simmons CP, Scott TW, Farrar JJ, and Hay SI. 2013. The global distribution and burden of dengue. Nature 496:504-507. 10.1038/nature12060

Bielejec F, Rambaut A, Suchard MA, and Lemey P. 2011. SPREAD: spatial phylogenetic reconstruction of evolutionary dynamics. Bioinformatics 27:2910-2912. 10.1093/bioinformatics/btr481 
411 Bouckaert R, Heled J, Kuhnert D, Vaughan T, Wu CH, Xie D, Suchard MA, Rambaut A, and

412

413

414

415

416

417

418

419

420

421

422

423

424

425

426

427

428

429

430

431

432

433

434

435

436

437

438

439

440

441

442

443

444

445

446

447

448

449

450

451

452

453

454

455

456

457

458

459
Drummond AJ. 2014. BEAST 2: a software platform for Bayesian evolutionary

analysis. PLoS Comput Biol 10:e1003537. 10.1371/journal.pcbi.1003537

CDC. 2015. Dengue. Available at http://www.cdc.gov/Dengue/ (accessed January 4 2015).

Chan M, and Johansson MA. 2012. The incubation periods of Dengue viruses. PLoS One 7:e50972. 10.1371/journal.pone.0050972

Chen R, and Vasilakis N. 2011. Dengue--quo tu et quo vadis? Viruses 3:1562-1608. $10.3390 / v 3091562$

Cori A, Ferguson NM, Fraser C, and Cauchemez S. 2013. A new framework and software to estimate time-varying reproduction numbers during epidemics. Am J Epidemiol 178:1505-1512. 10.1093/aje/kwt133

Cuong HQ, Vu NT, Cazelles B, Boni MF, Thai KT, Rabaa MA, Quang LC, Simmons CP, Huu TN, and Anders KL. 2013. Spatiotemporal dynamics of dengue epidemics, southern Vietnam. Emerg Infect Dis 19:945-953. 10.3201/eid1906.121323

Darriba D, Taboada GL, Doallo R, and Posada D. 2012. jModelTest 2: more models, new heuristics and parallel computing. Nat Methods 9:772. 10.1038/nmeth.2109

Delport W, Poon AF, Frost SD, and Kosakovsky Pond SL. 2010. Datamonkey 2010: a suite of phylogenetic analysis tools for evolutionary biology. Bioinformatics 26:2455-2457. 10.1093/bioinformatics/btq429

Drummond AJ, Ho SY, Phillips MJ, and Rambaut A. 2006. Relaxed phylogenetics and dating with confidence. PLoS Biol 4:e88. 10.1371/journal.pbio.0040088

du Plessis L, and Stadler T. 2015. Getting to the root of epidemic spread with phylodynamic analysis of genomic data. Trends Microbiol 23:383-386. 10.1016/j.tim.2015.04.007

Duong V, Lambrechts L, Paul RE, Ly S, Lay RS, Long KC, Huy R, Tarantola A, Scott TW, Sakuntabhai A, and Buchy P. 2015. Asymptomatic humans transmit dengue virus to mosquitoes. Proc Natl Acad Sci U S A. 10.1073/pnas.1508114112

Edgar RC. 2004a. MUSCLE: a multiple sequence alignment method with reduced time and space complexity. BMC Bioinformatics 5:113. 10.1186/1471-2105-5-113

Edgar RC. 2004b. MUSCLE: multiple sequence alignment with high accuracy and high throughput. 32 .

Edgar RC. 2010. Search and clustering orders of magnitude faster than BLAST. Bioinformatics 26:2460-2461. 10.1093/bioinformatics/btq461

Egger JR, Ooi EE, Kelly DW, Woolhouse ME, Davies CR, and Coleman PG. 2008. Reconstructing historical changes in the force of infection of dengue fever in Singapore: implications for surveillance and control. Bull World Health Organ 86:187-196. 10.2471/blt.07.040170

Endy TP. 2002. Epidemiology of Inapparent and Symptomatic Acute Dengue Virus Infection: A Prospective Study of Primary School Children in Kamphaeng Phet, Thailand. American Journal of Epidemiology 156:40-51. 10.1093/aje/kwf005

Guindon S, and Gascuel 0. 2003. A simple, fast, and accurate algorithm to estimate large phylogenies by maximum likelihood. Syst Biol 52:696-704.

$10.1080 / 10635150390235520$

Halstead SB. 2008. Dengue virus-mosquito interactions. Annu Rev Entomol 53:273-291. 10.1146/annurev.ento.53.103106.093326

Jeefoo P, Tripathi NK, and Souris M. 2011. Spatio-temporal diffusion pattern and hotspot detection of dengue in Chachoengsao province, Thailand. Int J Environ Res Public Health 8:51-74. 10.3390/ijerph8010051 
460 Kassim F. 2011. Use of dengue NS1 antigen for early diagnosis of dengue virus infection.

461

462

463

464

465

466

467

468

469

470

471

472

473

474

475

476

477

478

479

480

481

482

483

484

485

486

487

488

489

490

491

492

493

494

495

496

497

498

499

500

501

502

503

504

505

506

507 Southeast asian J trop Med public health 42.

Kosakovsky Pond SL, and Frost SD. 2005. Not so different after all: a comparison of methods for detecting amino acid sites under selection. Mol Biol Evol 22:12081222. $10.1093 / \mathrm{molbev} / \mathrm{msi} 105$

Kryazhimskiy S, and Plotkin JB. 2008. The population genetics of dN/dS. PLoS Genet 4:e1000304. 10.1371/journal.pgen.1000304

Lemey P, Rambaut A, Drummond AJ, and Suchard MA. 2009. Bayesian phylogeography finds its roots. PLoS Comput Biol 5:e1000520. 10.1371/journal.pcbi.1000520

Librado P, and Rozas J. 2009. DnaSP v5: a software for comprehensive analysis of DNA polymorphism data. Bioinformatics 25:1451-1452. 10.1093/bioinformatics/btp187

Maddison WP, and Maddison DR. 2014. Mesquite: a modular system for evolutionary analysis. . Version $3.01 \mathrm{ed}$ : http://mesquiteproject.org.

Mondini A, de Moraes Bronzoni RV, Nunes SH, Chiaravalloti Neto F, Massad E, Alonso WJ, Lazzaro ES, Ferraz AA, de Andrade Zanotto PM, and Nogueira ML. 2009. Spatiotemporal tracking and phylodynamics of an urban dengue 3 outbreak in Sao Paulo, Brazil. PLoS Negl Trop Dis 3:e448. 10.1371/journal.pntd.0000448

Nunes MR, Faria NR, Vasconcelos HB, Medeiros DB, Silva de Lima CP, Carvalho VL, Pinto da Silva EV, Cardoso JF, Sousa EC, Jr., Nunes KN, Rodrigues SG, Abecasis AB, Suchard MA, Lemey P, and Vasconcelos PF. 2012. Phylogeography of dengue virus serotype 4, Brazil, 2010-2011. Emerg Infect Dis 18:1858-1864. 10.3201/eid1811.120217

Nunes MR, Palacios G, Faria NR, Sousa EC, Jr., Pantoja JA, Rodrigues SG, Carvalho VL, Medeiros DB, Savji N, Baele G, Suchard MA, Lemey P, Vasconcelos PF, and Lipkin WI. 2014. Air travel is associated with intracontinental spread of dengue virus serotypes 1-3 in Brazil. PLoS Negl Trop Dis 8:e2769. 10.1371/journal.pntd.0002769

Poblap T, Nitatpattana N, Chaimarin A, Barbazan P, Chauvancy G, Yoksan S, and Gonzalez JP. 2006. Silent transmission of virus during a Dengue epidemic, Nakhon Pathom Province, Thailand 2001. Southeast asian J trop Med public health 37:899-903.

Pond SL, Frost SD, and Muse SV. 2005. HyPhy: hypothesis testing using phylogenies. Bioinformatics 21:676-679. 10.1093/bioinformatics/bti079

Raghwani J, Rambaut A, Holmes EC, Hang VT, Hien TT, Farrar J, Wills B, Lennon NJ, Birren BW, Henn MR, and Simmons CP. 2011. Endemic dengue associated with the co-circulation of multiple viral lineages and localized density-dependent transmission. PLoS Pathog 7:e1002064. 10.1371/journal.ppat.1002064

Rasmussen DA, Boni MF, and Koelle K. 2014. Reconciling phylodynamics with epidemiology: the case of dengue virus in southern Vietnam. Mol Biol Evol 31:258-271. 10.1093/molbev/mst203

Salje H, Lessler J, Endy TP, Curriero FC, Gibbons RV, Nisalak A, Nimmannitya S, Kalayanarooj S, Jarman RG, Thomas SJ, Burke DS, and Cummings DA. 2012. Revealing the microscale spatial signature of dengue transmission and immunity in an urban population. Proc Natl Acad Sci U S A 109:9535-9538. 10.1073/pnas.1120621109

Schreiber MJ, Holmes EC, Ong SH, Soh HS, Liu W, Tanner L, Aw PP, Tan HC, Ng LC, Leo YS, Low JG, Ong A, Ooi EE, Vasudevan SG, and Hibberd ML. 2009. Genomic 
508

509

510

511

512

513

514

515

516

517

518

519

520

521

522

523

524

525

526

527

528

529

530

531

532

533

534

535

536

537

538

539

540 epidemiology of a dengue virus epidemic in urban Singapore. J Virol 83:41634173. 10.1128/JVI.02445-08

Shepard DS, Coudeville L, Halasa YA, Zambrano B, and Dayan GH. 2011. Economic impact of dengue illness in the Americas. Am J Trop Med Hyg 84:200-207. 10.4269/ajtmh.2011.10-0503

Stadler T, Kuhnert D, Bonhoeffer S, and Drummond AJ. 2013. Birth-death skyline plot reveals temporal changes of epidemic spread in HIV and hepatitis $\mathrm{C}$ virus (HCV). Proc Natl Acad Sci U S A 110:228-233. 10.1073/pnas.1207965110

Stahl HC, Butenschoen VM, Tran HT, Gozzer E, Skewes R, Mahendradhata Y, RungeRanzinger S, Kroeger A, and Farlow A. 2013. Cost of dengue outbreaks: literature review and country case studies. BMC Public Health 13:1048. 10.1186/14712458-13-1048

Tajima F. 1989. Statistical method for testing the neutral mutation hypothesis by DNA polymorphism. Genetics 123:585-595.

Teixeira Mda G, Barreto ML, Costa Mda C, Ferreira LD, Vasconcelos PF, and Cairncross S. 2002. Dynamics of dengue virus circulation: a silent epidemic in a complex urban area. Trop Med Int Health 7:757-762.

Teixeira MG, Siqueira JB, Jr., Ferreira GL, Bricks L, and Joint G. 2013. Epidemiological trends of dengue disease in Brazil (2000-2010): a systematic literature search and analysis. PLoS Negl Trop Dis 7:e2520. 10.1371/journal.pntd.0002520

Vazquez-Prokopec GM, Kitron U, Montgomery B, Horne P, and Ritchie SA. 2010. Quantifying the spatial dimension of dengue virus epidemic spread within a tropical urban environment. PLoS Negl Trop Dis 4:e920. 10.1371/journal.pntd.0000920

Villabona-Arenas CJ, de Oliveira JL, Capra Cde S, Balarini K, Loureiro M, Fonseca CR, Passos SD, and Zanotto PM. 2014. Detection of four dengue serotypes suggests rise in hyperendemicity in urban centers of Brazil. PLoS Negl Trop Dis 8:e2620. 10.1371/journal.pntd.0002620

Villabona-Arenas CJ, and Zanotto PM. 2011. Evolutionary history of Dengue virus type 4: insights into genotype phylodynamics. Infect Genet Evol 11:878-885. 10.1016/j.meegid.2011.02.007 


\section{Figure 1 (on next page)}

Epidemiological situation in the Municipality of Guarujá.

A) Map of Guarujá and nearby localities; georeferenced samples are plotted over the map (See File S1 for displaying in Google Earth). B) Yearly dengue notifications for the State of São Paulo and some municipalities; dotted line intersects 2013. Fisher exact tests and corrections for multiple comparisons (Bonferroni and Benjamini-Hochberg) were used to assess the significance of the reduction in the number of cases from the previous epidemic year; tests were done by municipality $(n=429)$ and compared to the records for the State; Guarujá, São Vicente and São Paulo were among the municipalities $(n=103)$ with a significant reduction of notifications $(p<0.05)$. C) Monthly dengue notifications for the municipality of Guarujá during 2013. 
A

PeerJ

\section{Manuscript to be reviewed}

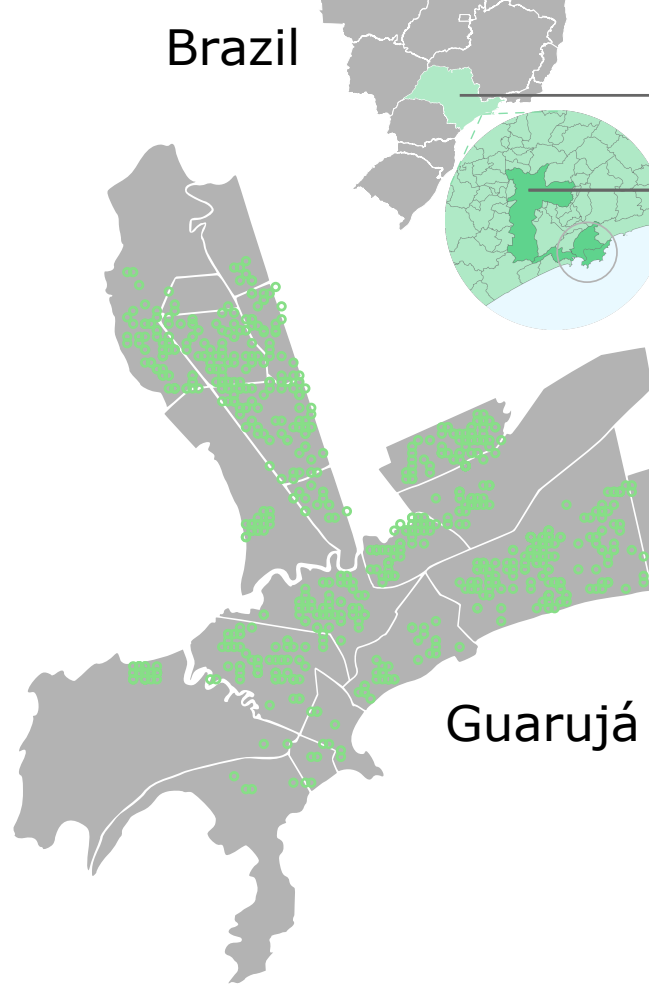

- São Paulo State

São Paulo

city
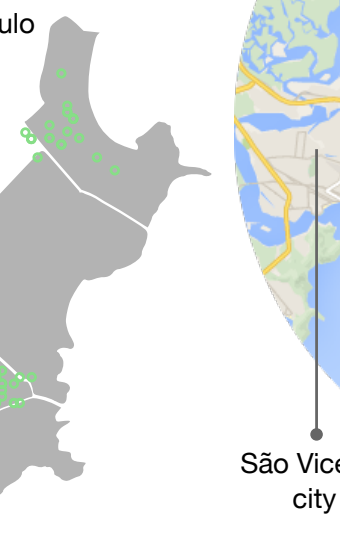

city

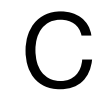

1000

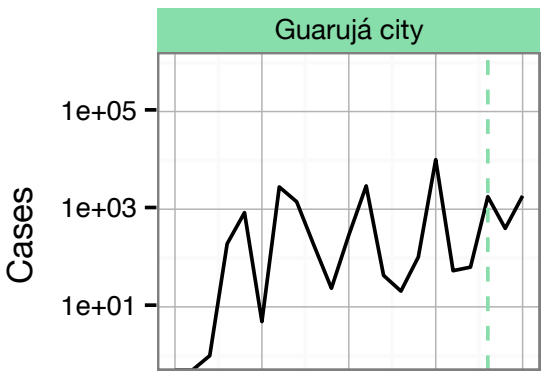

São Vicente city
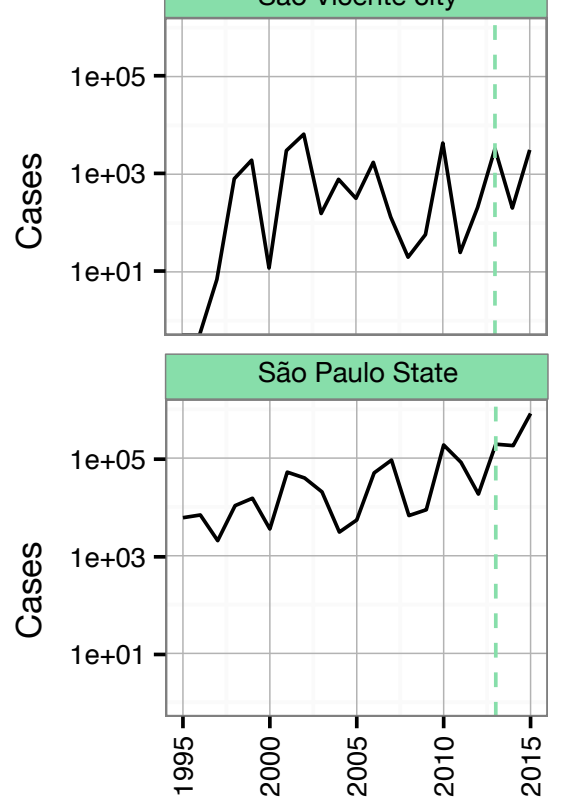

Peerj reviewing PDF | (2015:11:7786:1:0:NEW 9 Mar 2016)
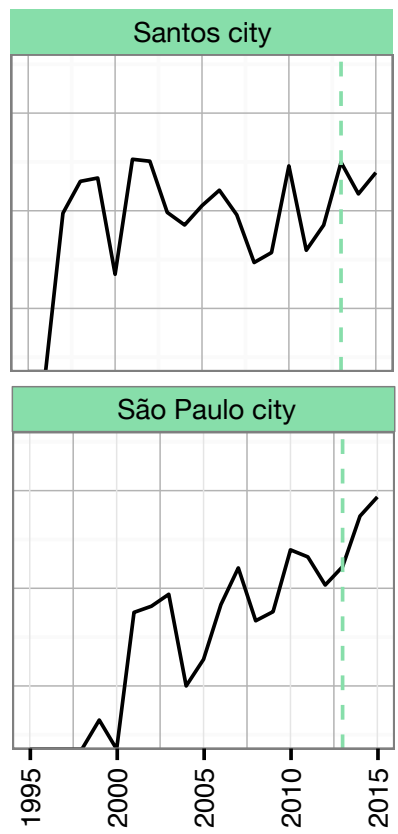

10

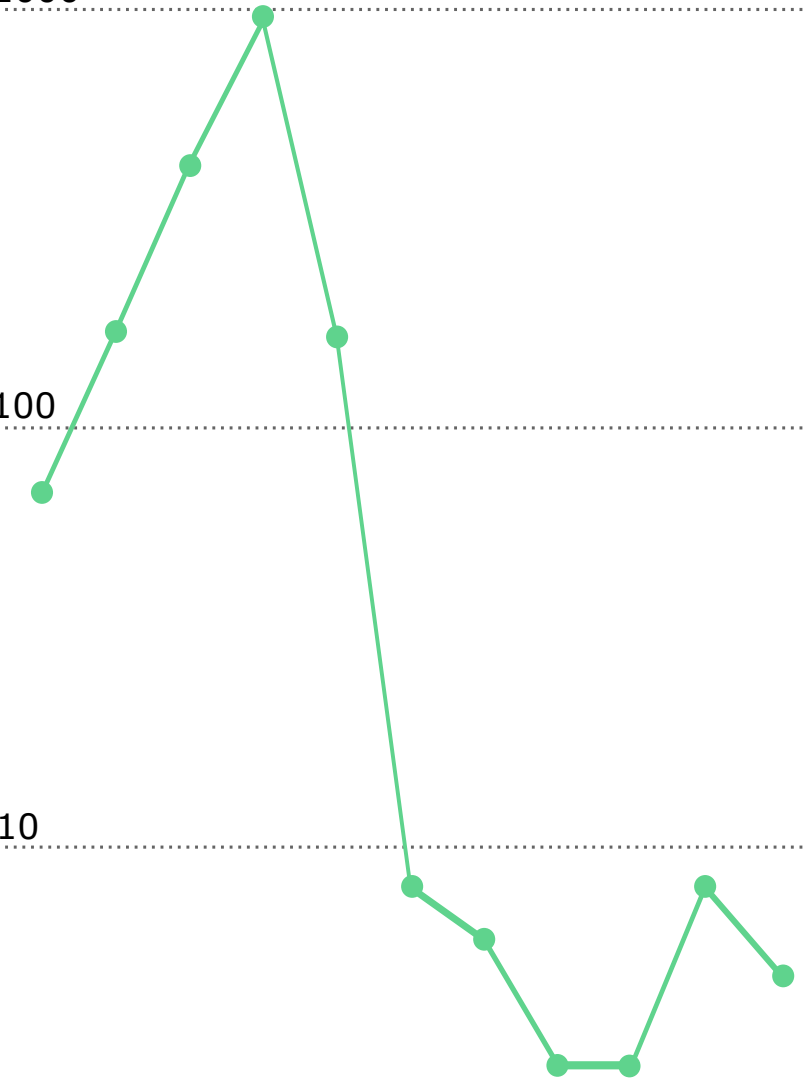

Jan Mar May Jul Sep Nov




\section{Figure 2 (on next page)}

Phylogenetic relationships and reproduction numbers of DENV-4 genotype II isolated in the municipality of Guarujá from January-June 2013.

A) Maximum clade credibility (MCC) tree inferred using envelope gene sequences. Branch tips were removed for simplicity. B) Median estimates and 95\% IC for the effective reproductive number using incidence time series data. For $B$ and $C$ official dengue reports done by epidemiological week and sampling done in a daily basis are presented. The band represents the period in which the epidemic alert was announced. The gray triangle informs when the neighboring city of Santos announced its own epidemic alert. 
PeerJ

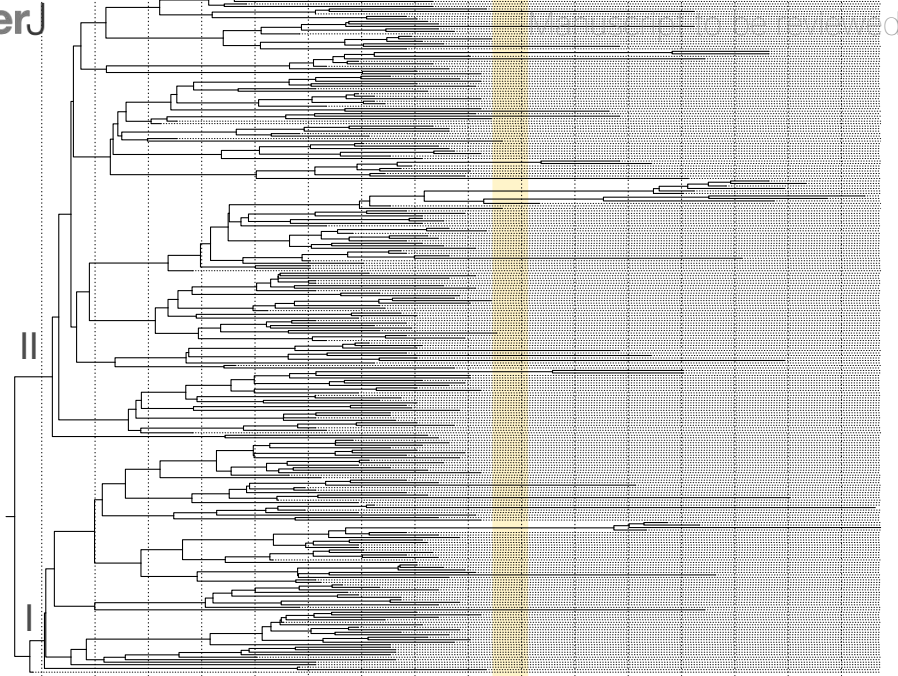

\begin{tabular}{llllllllllllll}
\hline 0.0 & 20.0 & 40.0 & 60.0 & 80.0 & 100.0 & 120.0 & 140.0 & 160.0
\end{tabular}

January
Tebruary
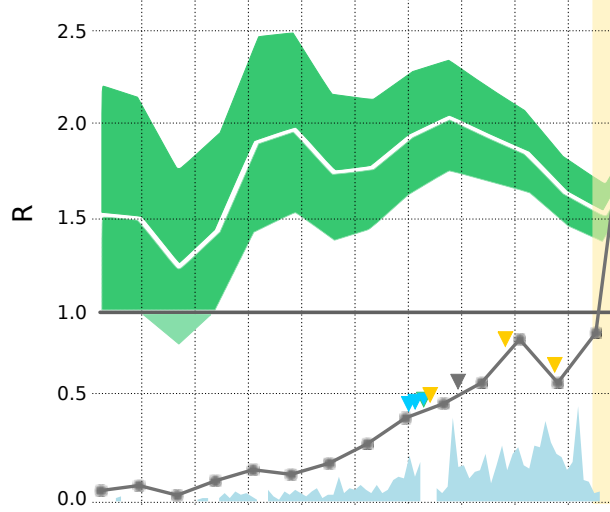

Source reduction

Peerj reviewing pof ${ }_{\text {Initiatives (2015:11:7786:1:0:NEW } 9 \text { Mar 2016) }}{ }^{2}$ campaigns shantytows in schools
Insecticide nebulization in Enseada, Morrinhos and Pae-Cará 
Figure 3 (on next page)

Diffusion of DENV-4 genotype II in the municipality of Guarujá from January-June 2013.

Discontinuous green areas represent discrete areas. A) Introduction routes into each area. B) Routes that best explain virus diffusion all over the city. The reconstruction was done following a location-annotated MCC tree available as Fig. 4. C) The main avenues and highways of Guarujá. Names are given for the areas that are quoted in the text. 


\section{Paecará}

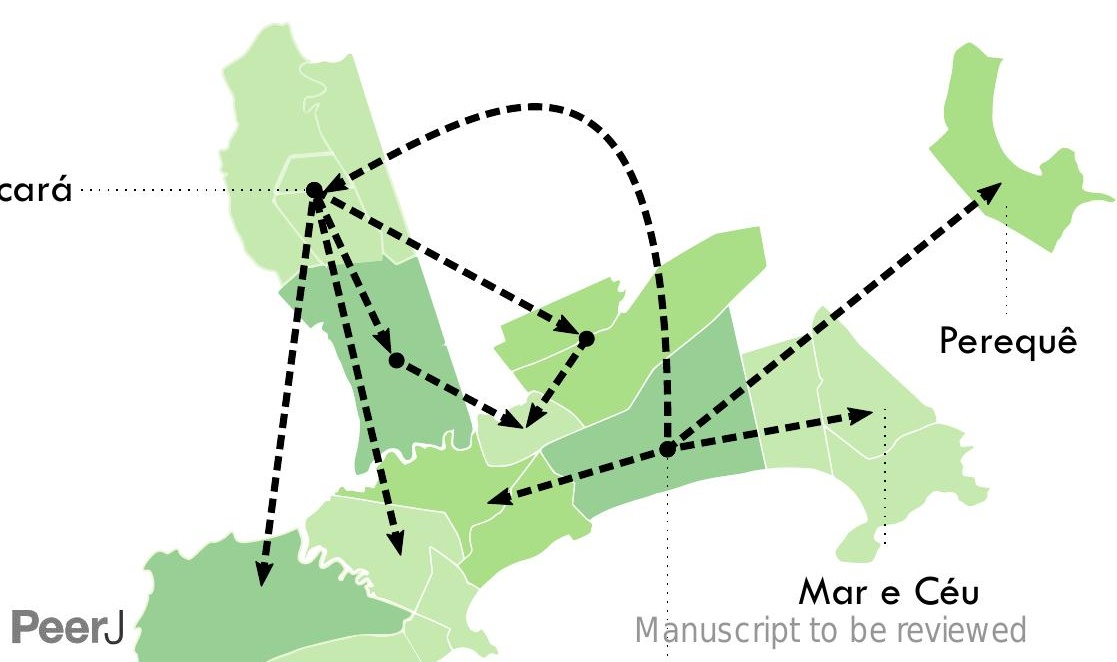

Enseada
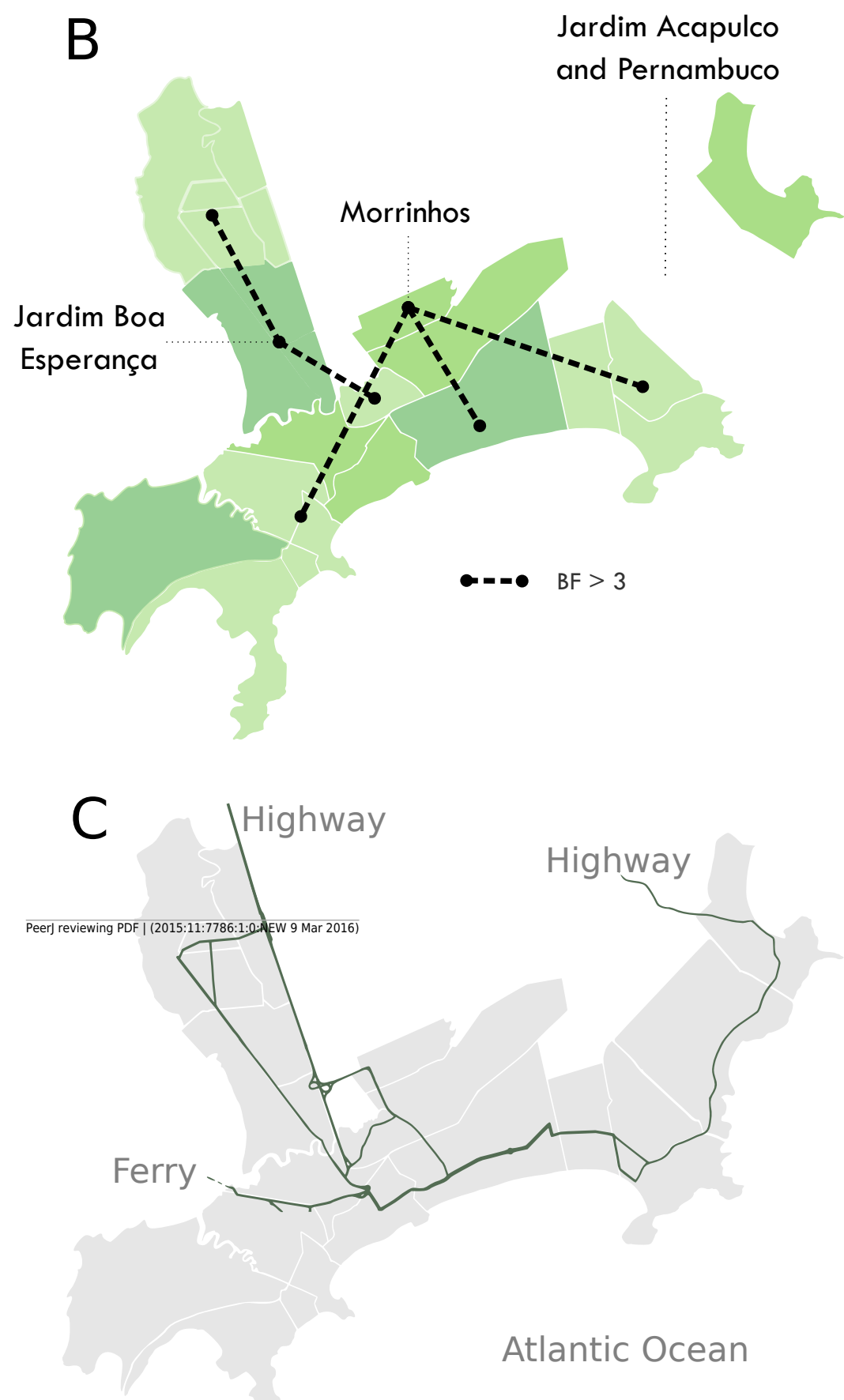
Figure 4 (on next page)

Location-annotated Maximum clade credibility tree.

Branch color corresponds to locations; tips were removed for simplicity. 
PeerJ

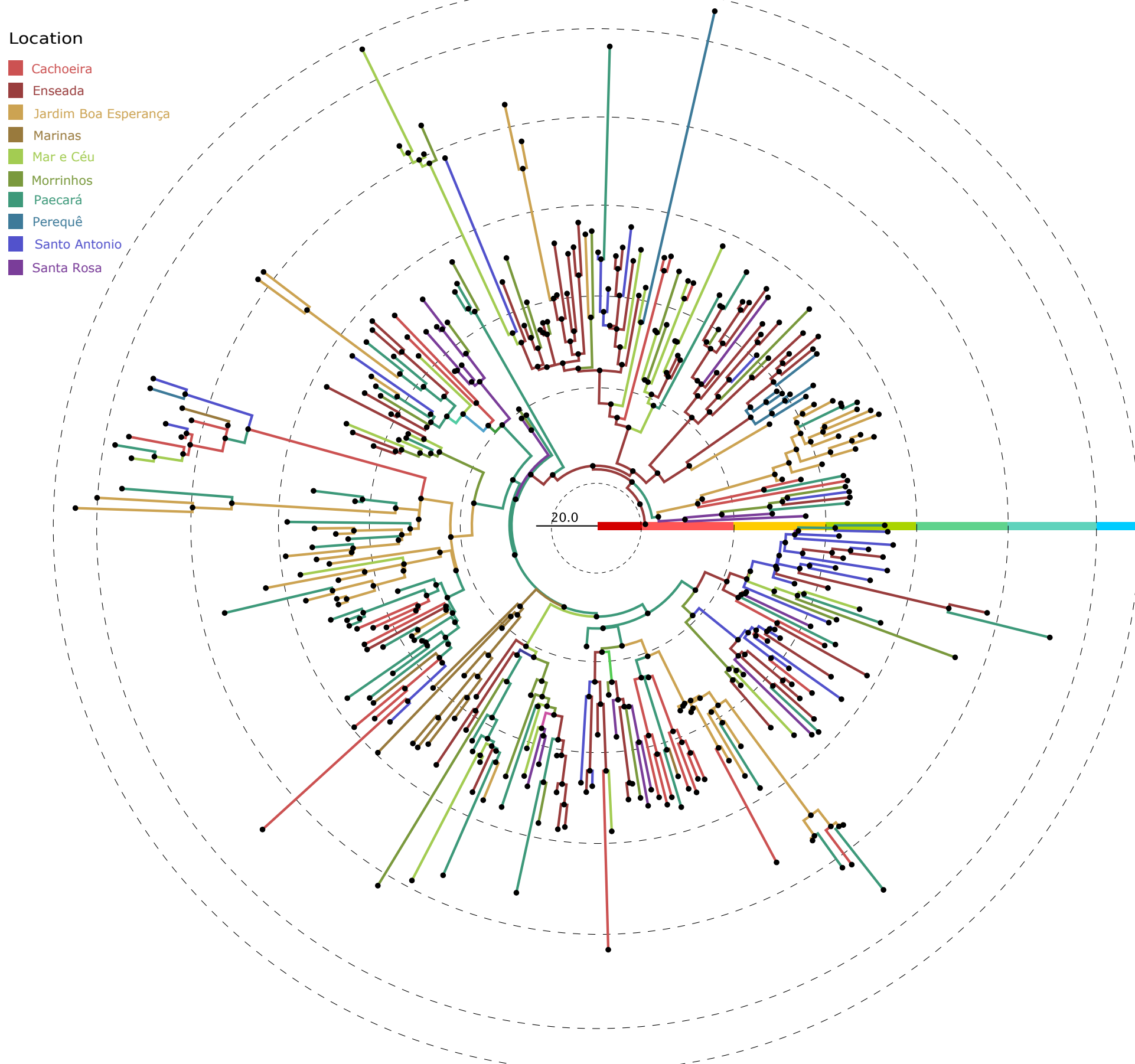

December January February March April May June 